University of Wollongong

Research Online

Faculty of Engineering and Information

Faculty of Engineering and Information

Sciences - Papers: Part A

Sciences

$1-1-2013$

Characterising poly (vinyl chloride)/Aliquat 336 polymer inclusion membranes: Evidence of phase separation and its role in metal extraction

Nurul-Syazana Abdul-Halim

University of Wollongong, nsbah996@uowmail.edu.au

Philip G. Whitten

University of Wollongong, whitten@uow.edu.au

Long D. Nghiem

University of Wollongong, longn@uow.edu.au

Follow this and additional works at: https://ro.uow.edu.au/eispapers

Part of the Engineering Commons, and the Science and Technology Studies Commons

Research Online is the open access institutional repository for the University of Wollongong. For further information contact the UOW Library: research-pubs@uow.edu.au 


\title{
Characterising poly (vinyl chloride)/Aliquat 336 polymer inclusion membranes: Evidence of phase separation and its role in metal extraction
}

\author{
Abstract \\ The miscibility of the base polymer poly (vinyl chloride) (PVC) and the extractant Aliquat 336 in polymer \\ inclusion membranes (PIMs) was investigated by characterisation of thermal transitions using differential \\ scanning calorimetry (DSC) and dynamic mechanical analysis (DMA). The extractions of Cd (II) and Zn (II) \\ using PVC/Aliquat 336 PIMs with different base polymer/extractant composition and different extraction \\ temperature were also investigated. Changes in the PIM's heat capacity measured by DSC were small, \\ thus, could only be used to determine the glass transition temperature (Tg) of PIMs with low Aliquat 336 \\ content. On the other hand, DMA results clearly identify the $(\mathrm{Tg})$ and melting temperature $(\mathrm{Tm})$ of \\ separate PVC and Aliquat 336 rich phases in the PIMs. Results reported here indicate that the PVC/ \\ Aliquat 336 PIMs are phase separated. This phase separation has important implications to the \\ extraction of target metallic ions by PIMs. Extraction studies showed that the extraction of metallic ions \\ occurred only when the proportion of Aliquat 336 in PIMs was about $30 \mathrm{wt} . \%$ or higher. The extraction rate \\ could be improved by increasing the temperature and thus the target ion transport in the Aliquat 336 \\ phase.

\section{Keywords} \\ its, role, metal, extraction, vinyl, chloride, aliquat, 336, characterising, polymer, poly, inclusion, membranes, \\ evidence, phase, separation

\section{Disciplines \\ Engineering | Science and Technology Studies}

\section{Publication Details} \\ Abdul-Halim, N., Whitten, P. G. \& Nghiem, L. D. (2013). Characterising poly (vinyl chloride)/Aliquat 336 \\ polymer inclusion membranes: Evidence of phase separation and its role in metal extraction. Separation \\ and Purification Technology, 119 (November), 14-18.
}




\title{
$1 \quad$ Characterising poly (vinyl chloride)/Aliquat 336 polymer 2 inclusion membranes: evidence of phase separation and its 3 role in metal extraction
}

4

5

6
Revised Manuscript Submitted to

Separation Purification Technology

August 2013

Nurul Syazana Abdul Halim ${ }^{1}$, Philip G. Whitten ${ }^{2}$ and Long D. Nghiem ${ }^{1, *}$

\author{
${ }^{1}$ Strategic Water Infrastructure Laboratory \\ School of Civil, Mining and Environmental Engineering \\ University of Wollongong, NSW 2522, Australia \\ ${ }^{2}$ School of Mechanical, Materials and Mechatronics \\ University of Wollongong, NSW 2522, Australia
}




\section{Abstract}

16 The miscibility of the base polymer poly (vinyl chloride) (PVC) and the extractant Aliquat

17336 in polymer inclusion membranes (PIMs) was investigated by characterisation of thermal

18 transitions using differential scanning calorimetry (DSC) and dynamic mechanical analysis

19 (DMA). The extractions of Cd (II) and Zn (II) using PVC/Aliquat 336 PIMs with different

20 base polymer/extractant composition and different extraction temperature were also

21 investigated. Changes in the PIM's heat capacity measured by DSC were small, thus, could

22 only be used to determine the glass transition temperature $\left(\mathrm{T}_{\mathrm{g}}\right)$ of PIMs with low Aliquat 336

23 content. On the other hand, DMA results clearly identify the $\left(\mathrm{T}_{\mathrm{g}}\right)$ and melting temperature

$24\left(\mathrm{~T}_{\mathrm{m}}\right)$ of separate PVC and Aliquat 336 rich phases in the PIMs. Results reported here indicate

25 that the PVC/Aliquat 336 PIMs are phase separated. This phase separation has important

26 implications to the extraction of target metallic ions by PIMs. Extraction studies showed that

27 the extraction of metallic ions occurred only when the proportion of Aliquat 336 in PIMs was

28 about 30 wt.\% or higher. The extraction rate could be improved by increasing the temperature

29 and thus the target ion transport in the Aliquat 336 phase.

30 Keywords: Polymer inclusion membranes (PIMs); Metal extraction; Poly (vinyl chloride)

31 (PVC); Aliquat 336; Phase separation. 


\section{Introduction}

34 The development of polymer inclusion membranes (PIMs) has increased rapidly over the last two decades as a potential alternative to the conventional solvent-solvent extraction process for metal ion recovery [1, 2]. PIM film consists of a polymer, an extractant and if necessary a plasticizer. Extractant is an essential component which function as a guest host specific molecule that provides selective membrane permeability for target species [3]. PIMs consisting of poly-vinyl chloride (PVC) and Aliquat 336 were first applied by James et al. [4] for the construction of ion selective electrodes more than four decades ago. Since then, PVC/Aliquat 336 PIMs have been one of the most studied PIM systems for the extraction of metallic ions from the aqueous phase. Previous studies have shown successful extraction of metal ions and small organic molecules using PVC based PIMs containing Aliquat 336 [510]. However, it is not yet clear whether the PVC/Aliquat 336 PIM is a solid homogenous solution or a two phase heterogeneous mixture.

The mechanism of facilitated transport in PIMs is still open to speculation given the lack of understanding about the nature of their homogeneity. For a solid solution, the metal ion with the aid of an extractant is transported through a polymer matrix. For a heterogeneous solid, the metal ion with the aid of extractant is transported through continuous channels within a polymer matrix. In some instances, a combination of these two extremes may occur.

In recent years, several studies have been conducted to investigate the homogeneity of PIMs. Through scanning electron microscopy analysis, $\mathrm{Xu}$ et al. [11] speculated that at above 30 wt.\% Aliquat 336, the interior structure of PVC/Aliquat 336 PIMs contained micro channels filled with Aliquat 336. They also showed that there exists a critical Aliquat 336 content in PIMs of 30 to $40 \mathrm{wt}$ \% for the transport of Cd (II) to occur. Although the critical Aliquat 336 content has been confirmed by several other studies [11, 12], their speculation about the existence of micro channel in PIMs has not been substantiated. In fact, it has been contradicted by a recent study by St John et al. [13] who employed high resolution synchrotron-based fourier-transform infrared (FTIR) spectroscopy and proton-induced X-ray emission microspectrometry ( $\mu$-PIXE) to study the homogeneity of PVC/Aliquat 336 PIMs. $\mu$-PIXE results reported by them showed that PVC based PIMs containing 10 to 40 wt.\% 62 Aliquat 336 are homogenous at the micro-scale which is comparable to the scale investigated by Xu et al. [11]. In this paper, PVC based PIMs were prepared with different Aliquat 336 concentrations. This work aims to determine whether the PIMs produced are a solid solution or mixture by 
application of differential scanning calorimetry (DSC) and dynamic mechanical analysis (DMA) techniques to characterise the thermal transitions. This approach clarifies the miscibility of PVC and Aliquat 336. Extraction of Cd (II) and Zn (II) were also investigated in order to observe any correlation between solid structure, thermal analysis and membrane function.

\section{Materials and Methods}

\subsection{Reagents}

All reagents were obtained from Sigma Aldrich, Australia. High molecular weight poly (vinyl chloride) (PVC) and Aliquat 336 (tricaprylylmethylammonium chloride) were used as the base polymer and extractant respectively. The weight-average molecular weight of this PVC is $80,000 \mathrm{~g} / \mathrm{mol}$. Aliquat 336 is a mixture of tri-alkyl methyl ammonium chloride salts produced from the methylation of Alamine 336, with the substituent alkyl chain length containing between 6 and 12 carbon atoms. HPLC grade tetrahydrofuran (THF) was used without any further purification. Cadmium (II) and zinc (II) solutions used in the membrane extraction experiments and for calibration purposes were prepared from $\mathrm{Cd}\left(\mathrm{NO}_{3}\right)_{2}$ and $\mathrm{Zn}\left(\mathrm{NO}_{3}\right)_{2}$ (analytical grade). Milli-Q grade water (Milipore, Australia) was used for the preparation of all aqueous solutions.

\subsection{Preparation of PVC/Aliquat 336 PIMs}

PIMs at different Aliquat 336 concentrations were prepared by dissolving Aliquat 336 and PVC in THF. Each mixture contains a combined Aliquat 336 and PVC weight of $600 \mathrm{mg}$. The volume of THF used was between 5 to $10 \mathrm{~mL}$ depending on the weight fraction of PVC. The mixtures were stirred vigorously for 1 hour resulting in a clear solution. The solution was then poured into a Petri dish with a diameter of $70 \mathrm{~mm}$ and covered with filter paper $(0.45 \mu \mathrm{m})$. The THF solvent was allowed to evaporate over about 48 hours forming a membrane. The membranes were peeled from the Petri dish and stored in the dry condition for further experiments. PVC films were prepared using the same protocol but without the addition of Aliquat 336.

\subsection{Extraction protocol}

Extraction experiments were conducted in bath mode [5, 6, 14]. Membranes were cut into small pieces of about $1 \mathrm{~cm}^{2}$ in area. The membrane pieces with an individual weight of approximately $0.55 \mathrm{~g}$ were placed in beakers containing $100 \mathrm{~mL}$ of extraction solution. The extraction solution contained $50 \mathrm{mg} / \mathrm{L}$ of either Cd (II) or Zn (II) in 1 M hydrochloric acid ( $\mathrm{HCl})$ and was placed in a temperature controlled water bath (Neslab RTE 7, Thermo 
Scientific Inc., Waltham, MA, USA). The solution was stirred continuously and $1 \mathrm{~mL}$ of

100 aliquot was taken at a specific time intervals for metal ion analysis using Atomic Adsorption 101 Spectrometry analysis (Varian SpectrAA 300 AAS, Australia). Calibration using standard Cd 102 (II) and Zn (II) solutions was conducted prior to each batch of analysis. The linear regression 103 coefficient for all calibration curves were greater than 0.98 .

2.4 Differential scanning calorimetry (DSC) analysis

DSC analysis for PVC/Aliquat 336 PIMs was carried out using a DSC Q-100 (TA Instrument, USA). The experiment was conducted at a heating rate of $10{ }^{\circ} \mathrm{C} / \mathrm{min}$ in the temperature range of -50 to $110^{\circ} \mathrm{C}$. Approximately $10 \mathrm{mg}$ of PVC/Aliquat $336 \mathrm{PIM}$ sample was used and encapsulated in standard aluminium pans while a hermetic pan was used for pure Aliquat 336 sample. Melting temperatures $\left(\mathrm{T}_{\mathrm{m}}\right)$ were reported based on the onset value. DMA Q 800 (TA Instrument, USA) was used to characterise the thermal transitions of PVC/Aliquat 336 PIMs. A film-clamp was used with a heating rate of $4{ }^{\circ} \mathrm{C} / \mathrm{min}$ over the temperature range of -100 to $180{ }^{\circ} \mathrm{C}$ at a frequency of $1 \mathrm{~Hz}$. The temperatures associated with transitions were identified by the peak in tan delta curve. For DMA, the thermal transitions were labelled in order from highest to lowest temperature.

\section{3. Results and discussion}

\section{$117 \quad 3.1$ Membrane characterization}

118 3.1.1 Thermal analysis

119 DSC analysis of the PVC as supplied showed a glass transition temperature $\left(\mathrm{T}_{\mathrm{g}}\right)$ of $85{ }^{\circ} \mathrm{C}$

120 (Figure 1) which is consistent with literature values [15, 16]. In contrast, DSC analysis of the 121 PVC cast from THF solution exhibited a $\mathrm{T}_{\mathrm{g}}$ of $63{ }^{\circ} \mathrm{C}$ (Figure 1). PIMs containing 10 to 40 122 wt.\% Aliquat 336 exhibited a $\mathrm{T}_{\mathrm{g}}$ in the range of 55 to $63{ }^{\circ} \mathrm{C}$ (Figure 2). PIMs containing 50 123 to $70 \mathrm{wt} . \%$ Aliquat 336 exhibited a $\mathrm{Tg}_{\mathrm{g}}$ that was too subtle for designation using the described 124 experimental procedure. DSC analysis of the supplied neat Aliquat 336 did not exhibit a $\mathrm{T}_{\mathrm{g}}$ 125 but exhibited a $\mathrm{T}_{\mathrm{m}}$ of $-19^{\circ} \mathrm{C}$ (Figure 1 ), which is also consistent with the report value of -20 $126{ }^{\circ} \mathrm{C}[17]$.

127 The DMA is another thermal analysis technique that is frequently employed to characterise 128 thermal transitions of polymers. The DMA isolates thermal transitions as substantial changes 129 in the storage modulus and a corresponding peak in the dissipation of energy $(\tan \delta)$. 
130 DMA results revealed that the PVC/Aliquat 336 PIMs contained one or two thermal 131 transitions with the number dependent on the fraction of Aliquat 336 (Figures 3-4). An $\alpha$ transition was observed at $71 \pm 8{ }^{\circ} \mathrm{C}$ for all Aliquat 336 concentrations studied here (Figure 2).

133 A $\beta$ transition was observed at $-18 \pm 1^{\circ} \mathrm{C}$ for PIMs samples containing 40 to $70 \mathrm{wt}$.\% Aliquat 336 (Figure 2). In addition, PIMs containing $10 \mathrm{wt}$ \% Aliquat 336 or more started to undergo degradation at about $100{ }^{\circ} \mathrm{C}$ as evidenced in an increase in the storage modulus (Figure 3).

\subsubsection{1 $\alpha$ transition}

141 The $\alpha$ transition is assigned to the $\mathrm{T}_{\mathrm{g}}$ of the PVC as it occurred in PVC cast from THF

142 without Aliquat 336 and it was consistent with DSC $T_{g}$ measurements (Figure 1). For the

143 PIMs membranes, the $\mathrm{T}_{\mathrm{g}}$ value coincides with the reduction in storage modulus from about $14410^{3} \mathrm{MPa}$ to less than $10 \mathrm{MPa}$ during heating (Figure 3). The PVC samples cast from THF 145 without Aliquat 336 were semi-crystalline at temperatures higher than the $\alpha$ transition. The 146 semi-crystalline nature of PVC the film (without any Aliquat 336) is evidenced by the 147 observation of a storage modulus higher than $1 \mathrm{MPa}$ at $100{ }^{\circ} \mathrm{C}$ (Figure 3).

148 For many plasticised polymers, the $\mathrm{T}_{\mathrm{g}}$ is typically a function of the plasticizer content 149 predicted by the Fox equation [18]:

$150 \quad \frac{1}{T_{g}}=\frac{w_{1}}{T_{g, 1}}+\frac{w_{2}}{T_{g, 2}}$ where $w_{1}$ and $w_{2}$ are weight fractions of components 1 and $2 . T_{g, 1}$ and $T_{g, 2}$ are the $T_{g}$ 's of components 1 and 2, and, $T_{g}$ is the single $T_{g}$ of the plasticized polymer.

153 This is because the plasticizer molecules form a solid solution with the polymer and enhance segmental mobility. For PVC based PIMs, Aliquat 336 has been widely reported to behave as a plasticizer $[2,10,19]$. For the PIMs observed here, the $T_{g}$ was independent of the Aliquat 336 concentration over the range of 0 to 70 wt.\%. In contrast, by using the Fox equation [18], the $\mathrm{T}_{\mathrm{g}}$ for a PIMs containing $70 \mathrm{wt} . \%$ Aliquat 336 was predicted to be $\leq 4{ }^{\circ} \mathrm{C}$ by assuming that the $\mathrm{T}_{\mathrm{g}}$ of the Aliquat 336 is about $\leq-20{ }^{\circ} \mathrm{C}$ [17], the $\mathrm{T}_{\mathrm{g}}$ of $\mathrm{PVC}$ is about $85^{\circ} \mathrm{C}$ and all of 
the Aliquat 336 is plasticizing the PVC. Hence, the Aliquat 336 is not solvating or depressing the $T_{g}$ of a substantial fraction of the PVC, and, is likely to be present in a separate phase.

161 Aliquat 336 is classified as a plasticiser for PVC as its incorporation transforms brittle PVC 162 into a plastic (a material capable of exhibiting substantial plastic deformation). Furthermore, the addition of Aliquat 336 also reduces the Young's modulus of PVC (also referred to as softening). The results presented here indicate that Aliquat 336 does not achieve the plasticization by forming a solid solution with PVC and depressing its $\mathrm{T}_{\mathrm{g}}$. The mechanism of plasticization may be the formation of a sponge like structure of PVC containing Aliquat 336 in the sponge pores.

The $\mathrm{T}_{\mathrm{g}}$ of the PVC cast from THF as determined by DMA and DSC $\left(68{ }^{\circ} \mathrm{C}\right.$ and $63{ }^{\circ} \mathrm{C}$ respectively) was well below that observed for PVC in the supplied condition determined by DSC $\left(85^{\circ} \mathrm{C}\right)$. This difference occurred in the absence of Aliquat 336, but after dissolution and drying from THF. It is likely that the $\mathrm{T}_{\mathrm{g}}$ depression observed after exposure to THF, results from a small amount of residual THF in the PVC. For example, $1 \mathrm{wt} . \%$ residual THF is sufficient to achieve a depression of $30^{\circ} \mathrm{C}$ [20].

\subsubsection{2 $\beta$ transition}

175 The $\beta$ transition is assigned to the melting temperature $\left(\mathrm{T}_{\mathrm{m}}\right)$ of the Aliquat 336 rich phase, as 176 it corresponds to the $\mathrm{T}_{\mathrm{m}}$ of neat Aliquat 336 observed by DSC (Figure 1). The 177 correspondence was confirmed where a piece of cloth soaked in Aliquat 336 subjected to 178 DMA exhibited a thermal transition over the same temperature range. The $\beta$ transition was independent of Aliquat 336 concentration for concentrations of 40 to $70 \mathrm{wt}$ \% as verified by a peak in the $\tan \delta$ indicating it's composition is constant (Figures 2 and 4). The storage modulus also shows a decrease at about $-22{ }^{\circ} \mathrm{C}$ for Aliquat 336 concentrations of 20 and 30 wt.\% indicating that they also contain an Aliquat 336 rich phase (Figure 3). There is no

183 indication of an Aliquat 336 rich phase for 10 wt.\% Aliquat 336 by DMA. Although the temperature where the $\beta$ transition occurred is independent of Aliquat 336 concentration, the storage modulus change associated with the $\beta$ transition increases with Aliquat 336 concentration.

\subsubsection{Membrane structure}

The thermal analysis data demonstrate that the PIMs membranes containing 20 to 70 wt.\% Aliquat 336 are a two phase structure containing a discrete Aliquat 336 rich phase, and a discrete PVC rich phase. However, thermal analysis provides no insights into whether the 
191 Aliquat 336 rich phase is continuous or in closed pores and it provides no insight into the

192 spatial dimensions of the phases. As the membrane structure is heterogeneous, and previous

193 reports have shown a threshold where heavy metals extraction begins, it is most likely that

194 the extraction are dominated by transport via a continuous or semi-continuous Aliquat 336

195 rich phase.

196 As Aliquat 336 and PVC form a solution with an appropriate concentration of THF, phase

197 separation may occur during the evaporation of THF when the membrane is formed.

198 Consequently, the phase morphology is determined by both the composition and fabrication

199 conditions of the PIMs. In other words, the shape, size and distribution of the second phase

200 are a function of the solvent evaporation rate and PIM thickness. Therefore, the structure of

201 the PIMs studied by St John et al. [13] and Xu et al. [11] may be different to that observed

202 here.

203

204

205

206

207

208

209

210

211

212

213

214

215

216

217

218

219

220

221

\subsection{Extraction experiments}

\subsubsection{Effect of Aliquat 336 content on metal ion extraction}

The extractions of Cd (II) and Zn (II) into PVC/Aliquat 336 PIMs are shown in Figures 5 and 6, respectively. In general, the extraction rate increased as the Aliquat 336 content in PIMs increased. When the extraction experiment was performed using PVC film that had been cast from THF without Aliquat 336, the metal ions extraction was found to be negligible, suggesting that the transport of both Cd (II) and Zn (II) were fulfilled by the extractant. Previously, PVC/Aliquat 336 PIMs have separated into two individual phases with one phase being rich in PVC ( $\alpha$ transition) and the other phase being rich in Aliquat 336 ( $\beta$ transition). For a heterogeneous PIM, the transport of ions across requires a continuous phase. Therefore, sufficient amount of extractant is essential to form continuous channels across the membrane for the extraction to occur. The threshold of Aliquat 336 content was observed to be $30 \mathrm{wt} \%$ where a major change was observed in both ion metals extractions (Figures 5 and 6). Below the percolation threshold, the extraction of PVC/Aliquat 336 PIMs was insignificant. Even though, the percolation threshold was attained at $30 \mathrm{wt} . \%$ of Aliquat 336, the extraction process was not completed even after 240 minutes. This result is in good agreement with the data reported by Xu et al. [11] that the extraction is not viable for PIMs containing less than 30 wt.\% of Aliquat 336. However, as Aliquat 336 content reaches 40 wt.\%, the extraction of Cd (II) and Zn (II) were almost completed (Figures 5 and 6) after 240 minutes. 
3.2.2 Effect of temperature on metal ions extraction

225

226

227

228

229

230

231

232

233

234

235

236

237

238

239

240

241

242

243

244

245

246

247

248

249

250

251

252

253

The influence of extraction temperature on the metal ions extraction was investigated for PIMs containing 30, 40 and 50 wt.\% of Aliquat 336. The extraction temperature of Cd (II) and Zn (II) from hydrochloric solution was varied at 10, 20, 35 and $50{ }^{\circ} \mathrm{C}$ and the results are plotted in Figures 7 and 8, respectively. The results show that extraction temperature has a significant effect on the extraction rate. At higher temperature, the extraction of Cd (II) and Zn (II) was 95\% completed, even though the Aliquat 336 content was only $30 \mathrm{wt} . \%$. The transport of metal ions through PIMs requires the diffusion of the metallic species itself or the metal-extractant complex through the membrane. An increase in temperature can increase not only the diffusion rate of metal-Aliquat 336 complex and Aliquat 336 molecules but also the rate of ion-exchange reaction between metal-Aliquat complexes. In either case, an increase in temperature will enhance the transport of the target metal ions in PIMs.

\section{[FIGURE 7]}

\section{[FIGURE 8]}

\section{Conclusion}

In the present study, the miscibility of the PVC/Aliquat 336 PIMs at various Aliquat 336 concentrations was investigated by measuring the $\mathrm{T}_{\mathrm{g}}$. The DMA has provided a distinct result for $\mathrm{T}_{\mathrm{g}}$ of PVC/Aliquat 336 PIMs and a $\mathrm{T}_{\mathrm{m}}$ of Aliquat 336. Results reported here indicate that PVC/Aliquat 336 PIMs were phase separated with two distinctive phases observed by DMA, that is an $\alpha$ transition at $71 \pm 8{ }^{\circ} \mathrm{C}$ and $\beta$ transition at $-18 \pm 1{ }^{\circ} \mathrm{C}$ where the later was not detected by DSC. Besides, the DMA results also suggest that Aliquat 336 is not a plasticizer since the $T_{g}$ did not decrease to below room temperature and is independent of Aliquat 336 content. However, an addition of Aliquat 336 has successfully produced transparent membranes with a flexible structure. It is noteworthy that, when more Aliquat 336 content was added, the PIMs become more flexible. Furthermore, both cadmium and zinc extraction occurred when Aliquat $336 \geq 30$ wt.\% which coincided with the appearance of a defined $\beta$ transition. The $\beta$ transition is assigned to the $T_{m}$ of an Aliquat 336 rich phase. In order to form this phase, Aliquat 336 content needs to be more than $30 \mathrm{wt}$ \%. The extraction rate also increases, as diffusivities in the Aliquat 336 phase increased which can be achieved by using higher extraction temperature. 


\section{References}

255

256

257

258

259

260

261

262

263

264

265

266

267

268

269

270

271

272

273

274

275

276

277

278

279

280

281

282

283

284

285

286

287

288

289

290

291

292

[1] Nghiem, L.D., Mornane, P., Potter, I.D., Perera, J.M., Cattrall, R.W., and Kolev, S.D., Extraction and transport of metal ions and small organic compounds using polymer inclusion membranes (PIMs). Journal of Membrane Science, 2006. 281(1-2): p. 7-41.

[2] Almeida, M.I.G.S., Cattrall, R.W., and Kolev, S.D., Recent trends in extraction and transport of metal ions using polymer inclusion membranes (PIMs). Journal of Membrane Science, 2012. 415-416(0): p. 9-23.

[3] Gardner, J.S., Walker, J.O., and Lamb, J.D., Permeability and durability effects of cellulose polymer variation in polymer inclusion membranes. Journal of Membrane Science, 2004. 229(1-2): p. 87-93.

[4] James, H., Carmack, G., and Freiser, H., Coated wire ion selective electrodes. Analytical Chemistry, 1972. 44(4): p. 856-857.

[5] Adelung, S., Lohrengel, B., and Nghiem, L.D., Selective transport of Cadmium by PVC/Aliquat 336 polymer inclusion membranes (PIMs): the role of membrane composition and solution chemistry. Membrane Water Treatment, 2012. 3(2): p. 123131.

[6] Upitis, A., Peterson, J., Nghiem, L.D., and Lukey, C., Metallic ion extraction using polymer inclusion membranes (PIMs): Optimising physical strength and extraction rate. Desalination and Water Treatment, 2009. 6(1-3): p. 41-47.

[7] Gherasim, C.V., Bourceanu, G., Olariu, R.I., and Arsene, C., A novel polymer inclusion membrane applied in chromium (VI) separation from aqueous solutions. Journal of Hazardous Materials, 2011. 197: p. 244-253.

[8] Kagaya, S., Ryokan, Y., Cattrall, R.W., and Kolev, S.D., Stability studies of poly(vinyl chloride)-based polymer inclusion membranes containing Aliquat 336 as a carrier. Separation and Purification Technology, 2012. 101: p. 69-75.

[9] O'Rourke, M., Cattrall, R.W., Kolev, S.D., and Potter, I.D., The Extraction and Transport of Organic Molecules Using Polymer Inclusion Membranes. Solvent Extraction Research and Evelopment -Japan, 2009. 16: p. 1-12.

[10] Gherasim, C.-V. and Bourceanu, G., Removal of chromium(VI) from aqueous solutions using a polyvinyl-chloride inclusion membrane: Experimental study and modelling. Chemical Engineering Journal, 2013. 220: p. 24-34.

[11] Xu, J.Y., Wang, L.J., Shen, W., Paimin, R., and Wang, X.G., The influence of the interior structure of Aliquat 336/PVC membranes to their extraction behavior. Separation Science and Technology, 2004. 39(15): p. 3527-3539.

[12] Wang, L., Paimin, R., Cattrall, R.W., Shen, W., and Kolev, S.D., The extraction of cadmium(II) and copper(II) from hydrochloric acid solutions using an Aliquat 336/PVC membrane. Journal of Membrane Science, 2000. 176(1): p. 105-111.

[13] St John, A.M., Best, S.P., Wang, Y.D., Tobin, M.J., Puskar, L., Siegele, R., Cattrall, R.W., and Kolev, S.D., Micrometer-Scale 2D mapping of the composition and 
homogeneity of polymer inclusion membranes. Australian Journal of Chemistry, 2011. 64(7): p. 930-938.

295

296

297

298

299

300

301

302

303

304

305

306

307

308

309

310

311

312

313

314

315

[14] Peterson, J. and Nghiem, L.D., Selective extraction of cadmium by polymer inclusion membranes containing PVC and Aliquat 336: Role base polymer and extractant. International Journal of Environmental Technology and Management, 2010. 12(2-4): p. 359-368.

[15] Elicegui, A., Val, J.J.d., Bellenger, V., and Verdu, J., A study of plasticization effects in poly(vinyl chloride). Polymer, 1997. 38(7): p. 1647-1657.

[16] Gomez Ribelles, J.L., Diaz-Calleja, R., Ferguson, R., and Cowie, J.M.G., Glass transition and physical ageing in plasticized poly(vinyl chloride). Polymer, 1987. 28: p. 2262-2266.

[17] Mikkola, J.-P., Virtanen, P., and Sjoholm, R., Aliquat 336[registered sign]-a versatile and affordable cation source for an entirely new family of hydrophobic ionic liquids. Green Chemistry, 2006. 8(3): p. 250-255.

[18] Fox, T.G., Influence of diluent and of copolymer composition on the glass temperature of a polymer system. Bulletin of the American Physical Society, 1956. 1(2): p. 123-128.

[19] Argiropoulos, G., Cattrall, R.W., Hamilton, I.C., Kolev, S.D., and Paimin, R., The study of a membrane for extracting gold(III) from hydrochloric acid solutions. Journal of Membrane Science, 1998. 138(2): p. 279-285.

[20] Adachi, K. and Ishida, Y., Effects of diluent on molecular motion and glass transitions in polymers - 2. The system polyvinylchloride-tetrahydrofuran. Journal of Polymer Science, Polymer Physics Edition, 1976. 14(12): p. 2219-2230. 